\title{
Melittin Induced G1 Cell Cycle Arrest and Apoptosis in Chago-K1 Human Bronchogenic Carcinoma Cells and Inhibited the Differentiation of THP-1 Cells into Tumour- Associated Macrophages
}

\author{
Chartsiam Tipgomut $^{1}$, Arin Wongprommoon ${ }^{2}$, Emi Takeo $^{3}$, Teeranai Ittiudomrak ${ }^{4}$, \\ Songchan Puthong ${ }^{5}$, Chanpen Chanchao ${ }^{1 *}$
}

\begin{abstract}
Background: Bronchogenic carcinoma (lung cancer) is one of the leading causes of death. Although many compounds isolated from natural products have been used to treat it, drug resistance is a serious problem, and alternative anti-cancer drugs are required. Here, melittin from Apis mellifera venom was used, and its effects on bronchogenic carcinoma cell proliferation and tumour-associated macrophage differentiation were evaluated. Methods: The half maximal inhibitory concentration (IC50) of melittin was measured by MTT. Cell death was observed by annexin V and propidium iodide (PI) co-staining followed by flow cytometry. Cell cycle arrest was revealed by PI staining and flow cytometry. To investigate the tumour microenvironment, differentiation of circulating monocytes (THP-1) into tumour-associated macrophages (TAMs) was assayed by sandwich-ELISA and interleukin (IL)-10 levels were determined. Cell proliferation and migration was observed by flat plate colony formation. Secretion of vascular endothelial growth factor (VEGF) was detected by ELISA. The change in expression levels of CatS, Bcl-2, and MADD was measured by quantitative RT-PCR. Results: Melittin was significantly more cytotoxic $(p<0.01)$ to human bronchogenic carcinoma cells (ChaGo-K1) than to the control human lung fibroblasts (Wi-38) cells. At $2.5 \mu \mathrm{M}$, melittin caused ChaGo-K1 cells to undergo apoptosis and cell cycle arrest at the G1 phase. The IL-10 levels showed that melittin significantly inhibited the differentiation of THP-1 cells into TAMs $(p<0.05)$ and reduced the number of colonies formed in the treated ChaGo-K1 cells compared to the untreated cells. However, melittin did not affect angiogenesis in ChaGo-K1 cells. Unlike MADD, Bcl-2 was up-regulated significantly $(\mathrm{p}<0.05)$ in melittin-treated ChaGo-K1 cells. Conclusion: Melittin can be used as an alternative agent for lung cancer treatment because of its cytotoxicity against ChaGo-K1 cells and the inhibition of differentiation of THP-1 cells into TAMs.
\end{abstract}

Keywords: Apoptosis- bronchogenic carcinoma- melittin- tumor-associated macrophage- cathepsin S

Asian Pac J Cancer Prev, 19 (12), 3427-3434

\section{Introduction}

Bronchogenic carcinoma (lung cancer) is one of the most common fatal cancers (Torre et al., 2015; Hasan et al., 2018). The high mortality in lung cancer patients is, due to the poor rate of early diagnosis, since patients are usually asymptomatic until the advanced stages, and due to the resistance to chemotherapy, which has become a severe problem (Felip and Rosell, 2007; Liang et al., 2018). This resistance is not only due to cellular mechanisms, but is also influenced by the tumour microenvironment, where many other components aid the survival of cancer cells (da Silva-Diz et al., 2018). Cells within this tumour microenvironment can be divided into two broad groups, one comprises immune cells, such as granulocytes, lymphocytes, and macrophages and the other comprises mesenchymal cells, such as endothelial cells and fibroblasts (Kerkar and Restifo, 2012). Macrophages can be classified into two distinct phenotypes, M1 and M2. The expression of CD38, G-protein coupled receptor 18 (Gpr 18), and formyl peptide receptor 2 (Fpr 2 ) genes is unique to M1 macrophages, while expression of early growth response protein 2 (Egr2) and cMyc is unique to M2 macrophages (Jablonski et al., 2015). Additional markers for M1 macrophages like iNOS, IL-6, SOSC3, and TNF- $\alpha$ and ARG1 and CCL24 for

${ }^{1}$ Department of Biology, ${ }^{4}$ Program in Biotechnology, Faculty of Science, 'Institute of Biotechnology and Genetic Engineering, Chulalongkorn University, 254 Phayathai Road, Bangkok, Thailand, ${ }^{2}$ Homerton College, University of Cambridge, Hills Road, Cambridge CB2 8PH, UK, ${ }^{3}$ Department of Biotechnology, Graduate School of Engineering, Osaka University, 2-1 Yamadaoka, Suita, Osaka, Japan.*For Correspondence: chanpen.c@chula.ac.th 
M2 macrophages have been reported (Qin et al., 2017). Furthermore, the expression pattern of interleukin (IL)10 , IL-12, transforming growth factor $\beta 1$, and TNF- $\alpha$ is important for investigating M1/M2 polarization (Mohammadi et al., 2017a). These mesenchymal cells play an important role in cell support and homeostasis. Furthermore, the surrounding extracellular matrix gives strength and flexibility to the tumour (Thanee et al., 2012). Cancer cells in a tumour microenvironment secret cytokines, chemokines, and growth factors for cancer cell proliferation and metastasis (Wong and Chang, 2018).

An important mechanism in the tumour microenvironment is the activation of circulating monocytes to enter the tumour, where they then differentiate and become tumour-associated macrophages (TAMs) under the influence of IL-4 and IL-10 secreted by the cancer cells (Pilling et al., 2017; Shao et al., 2016). Overall, TAMs may be a major component of the tumour microenvironment and are directly involved in tumour growth, infiltration, and metastasis. In addition, TAMs can suppress the immune functions of the cancer cells, promoting drug and radiation resistance (Shao et al., 2016; Zhang et al., 2016).

Conventional anti-cancer drugs frequently become ineffective due to chemoresistance, leading to invasion, metastasis, angiogenesis, and inflammation (Konrad et al., 2017). Thus, it is essential to find a newer compound to inhibit lung cancer cells and the differentiation of macrophages to TAMs.

For many years, bee venom has been used in apitherapy. Although, it is composed of a diverse range of proteins and peptides, melittin is the major protein and comprises about $50 \%(\mathrm{w} / \mathrm{w})$ of dry bee venom (Gajski and Garaj-Vrhovac, 2013). Melittin has anti-analgesic, anti-inflammatory, and antimicrobial activities (Gajski and Garaj-Vrhovac, 2013; Lin et al., 2017; Rady et al., 2017; Shi et al., 2016). However, no reports of melittin on lung cancer proliferation and monocyte differentiation are available. Here, the in vitro cytotoxicity of melittin against the human bronchogenic carcinoma (ChaGo-K1), human lung fibroblast (Wi-38), and human monocytic leukaemia (THP-1) cell lines was tested. Cell death and the changes in cell cycle arrest in melittin-treated ChaGo-K1 cells was evaluated in comparison to the Wi-38 cells. Additionally, the effect of melittin on differentiation of monocytes, in vitro cell migration, colony formation, and down-regulation of vascular endothelial growth factor (VEGF) levels involved in angiogenesis, were evaluated. Finally, the changes in gene expression levels of cathepsin S (CatS), B-cell lymphoma-2 (Bcl-2), and mitogen activating protein-kinase activating death domain (MADD) were reported.

\section{Materials and Methods}

\section{Chemicals}

Melittin, phorbol 12-myristate 13-acetate (PMA), and propidium iodide (PI) were purchased from Sigma-Aldrich Co. (MO, USA; catalogue no. M2272, P3139, and CP4864, respectively). Minimum essential medium (MEM), RPMI 1640 medium, foetal bovine serum
(FBS), and non-essential amino acids were purchased from Biochrom Ltd (Cambridge, UK) (catalogue no. FG0325, T121, S0415, and KO293, respectively). Annexin V-Alexa Fluor ${ }^{\circledR} 488$ conjugate was purchased from Thermo Fisher Scientific Inc. (MA, USA) (catalogue no. A13201). The human IL-10 enzyme-linked immunosorbent assay (ELISA) kit was purchased from Abcam PLC (Cambridge, UK) (catalogue no. ab46034). Human recombinant IL-13 and IL-4 were purchased from Preprotech Co. (NJ, USA) (catalogue no. 20013 and 20004 respectively), while the VEGF Human BioAssay ${ }^{\mathrm{TM}}$ ELISA Development Kit was purchased from US Biological Life Sciences (MA, USA) (catalogue no. 145985).

\section{Cell culture}

The ChaGo-K1, Wi-38, and THP-1 cell lines were obtained from Institute of Biotechnology and Genetic Engineering, Chulalongkorn University. The ChaGo-K1 and THP-1 cells were maintained in CM-R (RPMI 1640 medium supplemented with $10 \%$ (v/v) FBS, 1,000 $\mathrm{U} / \mathrm{mL}$ penicillin, $1.7 \mathrm{mM}$ streptomycin, and $2.7 \mu \mathrm{M}$ Fungizone $\left.^{\mathrm{TM}}\right)$, while Wi-38 cells were maintained in CM-M (MEM supplemented with 1\% (w/v) non-essential amino acids, $1 \mathrm{mM}$ sodium pyruvate, $10 \%$ (v/v) FBS, 1,000 U/mL penicillin, $1.7 \mathrm{mM}$ streptomycin, and 2.7 $\mu \mathrm{M}$ Fungizone ${ }^{\mathrm{TM}}$ ).

\section{Melittin cytotoxicity assay}

ChaGo-K1 and Wi-38 cells were suspended in CM-R and CM-M, respectively, at a concentration of 105 cells/ well and seeded at $200 \mu \mathrm{L} /$ well in 96-well culture plates. After an overnight incubation at $37^{\circ} \mathrm{C}$ in a $5 \%(\mathrm{v} / \mathrm{v}) \mathrm{CO}_{2}$ atmosphere, the media were supplemented with melittin at a final concentration of 7, 0.7, 0.007, 0.0007, and $0 \mu \mathrm{M}$ and cultured for 24,48 , and $72 \mathrm{~h}$ at $37^{\circ} \mathrm{C}$ with $5 \%(\mathrm{v} / \mathrm{v})$ $\mathrm{CO}_{2}$. Thereafter, $0.12 \mu \mathrm{M}$ 3-(4,5-dimethylthiazol-2-yl)2,5-diphenyltetrazolium bromide (MTT) was added and the cells were incubated for another $4 \mathrm{~h}$ before the culture medium was replaced with $150 \mu \mathrm{L}$ dimethylsufoxide and the absorbance at $540 \mathrm{~nm}$ (A540) was measured using a Multiskan $^{\mathrm{TM}}$ FC microplate photometer (Thermo Fisher Scientific Inc., MA, USA). The percentage of viable cells relative to control was calculated as show below:

Relative cell survival $($ in $\%)=\left(\mathrm{A}_{540}\right.$ of sample $\left.\times 100\right)$ $/\left(\mathrm{A}_{540}\right.$ of control)

A graph of the relative cell survival (in\%) against the concentration of melittin was plotted to derive the $\mathrm{IC}_{50}$ and $\mathrm{IC}_{70}$.

\section{Programmed cell death}

ChaGo-K1 cells were suspended in CM-R medium and seeded at $10^{6}$ cells/flask in a $25 \mathrm{~mL}$ flat-sided cell culture flask. Five groups of cells were prepared: (i) unstained cells, (ii) stained cells, stained cells treated with melittin at a final concentration of (iii) $0.7 \mu \mathrm{M}$ (IC50) and (iv) $2.5 \mu \mathrm{M}\left(\mathrm{IC}_{70}\right)$, and (v) stained cells treated with $0.9 \mu \mathrm{M}$ doxorubicin. After treatment, the cells were incubated for $24 \mathrm{~h}$ at $37^{\circ} \mathrm{C}$ with $5 \%(\mathrm{v} / \mathrm{v}) \mathrm{CO}_{2}$, then harvested, washed twice in $1 \mathrm{~mL}$ cold phosphate-buffered saline of $\mathrm{pH} 7.4$ 
(PBS), and resuspended in $50 \mu \mathrm{L}$ of $1 \times$ binding buffer $(10$ mM HEPES, pH 7.4, $140 \mathrm{mM} \mathrm{NaCl}$, and $2.5 \mathrm{mM} \mathrm{CaCl}_{2}$ ). Except for the unstained group, the cells were then stained with $1 \mu \mathrm{L}$ annexin V-FITC Alexa Fluor ${ }^{\circledR} 488$ and 0.004 $\mu \mathrm{M}$ PI solution at room temperature in the dark for 30 min prior to flow cytometric analysis using a FC $500 \mathrm{MPL}$ cytometer (Beckman Coulter Inc., CA, USA).

\section{Cell cycle analysis}

ChaGo-K1 cells were suspended in CM-R and seeded at $10^{6}$ cells/flask in a $25 \mathrm{~mL}$ flat-sided cell culture flask. Four groups of cells were prepared: (i) untreated cells, melittin-treated cells at a final concentration of (ii) $0.7 \mu \mathrm{M}$ ( $\mathrm{IC}_{50}$ ) and (iii) $2.5 \mu \mathrm{M}\left(\mathrm{IC}_{70}\right.$ ), and (iv) $0.9 \mu \mathrm{M}$ doxorubicin treated cells and were incubated for $24 \mathrm{~h}$ at $37^{\circ} \mathrm{C}$ with $5 \%(\mathrm{v} / \mathrm{v}) \mathrm{CO}_{2}$ and then harvested and washed with 1 $\mathrm{mL}$ cold PBS. The cells were then fixed with $1 \mathrm{~mL} 70 \%$ (v/v) ethanol at $-20^{\circ} \mathrm{C}$ overnight. Ethanol was removed, the cells were washed in $1 \mathrm{~mL}$ cold PBS, resuspended in $250 \mu \mathrm{L}$ cold PBS with $0.5 \mathrm{U}$ of RNase A, and incubated at $37^{\circ} \mathrm{C}$ for $30 \mathrm{~min}$. The cells were then centrifuged, resuspended in a $37.5 \mu \mathrm{L}$ PBS and $0.02 \mu \mathrm{M}$ PI solution, and incubated at room temperature in the dark for $30 \mathrm{~min}$ prior to flow cytometric analysis.

\section{Monocyte-to-macrophage differentiation}

THP-1 derived macrophages: THP-1 cells were cultured at $5 \times 10^{5}$ cells/well in 24-well culture plates in CM-R supplemented with $200 \mathrm{nM}$ PMA for $72 \mathrm{~h}$ at $37^{\circ} \mathrm{C}$ in a $5 \%(\mathrm{v} / \mathrm{v}) \mathrm{CO}_{2}$ atmosphere to induce differentiation. The differentiated cells displayed a cellular morphology similar to macrophages and adhered to the culture plates. Cells were treated with melittin at a final concentration of 0 (control), $0.044,0.088,0.175$, and $0.350 \mu \mathrm{M}$ for $24 \mathrm{~h}$ at $37{ }^{\circ} \mathrm{C}$ with $5 \%(\mathrm{v} / \mathrm{v}) \mathrm{CO}_{2}$. As control, PBS was added to the PMA-primed THP-1 cells, which is similar to Mohammadi et al., (2017b). The culture medium was then harvested and screened for IL-10 using the human IL-10 ELISA kit.

M2 polarized macrophages: The same method was followed for the preparation of THP-1-derived M2 macrophages. After melittin treatment, the cells were incubated for $24 \mathrm{~h}$ at $37^{\circ} \mathrm{C}$ with $5 \%(\mathrm{v} / \mathrm{v}) \mathrm{CO}_{2}$, but with the addition of IL-4 (25 ng/mL) and IL-13 $(25 \mathrm{ng} / \mathrm{mL})$ for a further $24 \mathrm{~h}$ to induce differentiation to M2 polarized macrophages. IL-10 levels in the medium were measured using the human IL-10 ELISA kit.

\section{Colony formation}

ChaGo-K1 cells were suspended in CM-R at 103 cells/well in a 6 -well plate and incubated at $37^{\circ} \mathrm{C}$ in a
$5 \%(\mathrm{v} / \mathrm{v}) \mathrm{CO}_{2}$ atmosphere in the presence of melittin at a final concentration of $0,0.175,0.35,0.7,1.4$, and 2.8 $\mu \mathrm{M}$ for $24 \mathrm{~h}$. The cells were then washed twice with cold PBS and incubated in $9 \mathrm{~mL}$ of CM-R for $14 \mathrm{~d}$ at $37^{\circ} \mathrm{C}$ in a $5 \%(\mathrm{v} / \mathrm{v}) \mathrm{CO}_{2}$ atmosphere to allow colony formation. The colonies were washed with cold PBS, fixed in $4 \%(\mathrm{v} / \mathrm{v})$ neutral-buffered formalin for $10 \mathrm{~min}$ and then stained by crystal violet. The number of colonies and their sizes were measured under light microscopy.

\section{Secretion of VEGF}

ChaGo-K1 cells were suspended in CM-R at $5 \times 10^{5}$ cells/well in a 6 -well plate and incubated with 0 or 0.7 $\mu \mathrm{M}$ of melittin at $37^{\circ} \mathrm{C}$ in a $5 \%(\mathrm{v} / \mathrm{v}) \mathrm{CO}_{2}$ atmosphere for $24 \mathrm{~h}$. The culture medium was then harvested and the concentration of VEGF was measured using the VEGF Human BioAssay ${ }^{\mathrm{TM}}$ ELISA Development Kit.

\section{Gene expression}

Total RNA was isolated using the RNeasy ${ }^{\circledR}$ Mini Kit (Cat\# 74104, Qiagen, Valencia, CA, USA). Concentration and purity of extracted total RNA was measured by the absorbance at 260 and $280 \mathrm{~nm}$. Quantitative reverse transcriptase polymerase chain reaction (RT-qPCR) was used to amplify CatS, Bcl-2, MADD and B-actin mRNA using the One Step SYBR PrimeScript RT-qPCR Kit II (Takara, Tokyo, Japan) as per the manufacturer's protocol. Respective forward and reverse primers of CatS, including the optimal condition of RT-qPCR were obtained from Zhang et al. (2016), while those of B-actin, Bcl-2 and MADD, including the optimal condition of RT-qPCR, were obtained from Buahorm et al. (2015). The relative expression levels of target genes were normalized to the expression level of the $\beta$-actin gene as control.

\section{Statistical analysis}

Each assay was performed in triplicates and the results are presented as mean \pm 1 standard deviation (SD). Data were analysed by ANOVA, while the significance of differences between means was ascertained by Tukey's-b or Duncan tests. Data was considered to be statistically significant at $\mathrm{p}<0.05$.

\section{Results}

\section{Cytotoxicity of melittin}

Melittin could inhibit the proliferation of both ChaGo-K1 and Wi-38 cells (Figure 1), with an $\mathrm{IC}_{50}$ of $0.79 \pm 0.02$ and $1.91 \pm 0.10 \mu \mathrm{M}$, respectively, during $24 \mathrm{~h}$ incubation (Figure 1A). Melittin $\mathrm{IC}_{50}$ value did not significantly differ when the incubation period

Table 1. Effect of Melittin and Doxorubicin (Positive Control) on Programmed Cell Death of ChaGo-K1 Cells

\begin{tabular}{lcccc}
\hline Treatment & Viable cells & Early apoptosis & Late apoptosis & Necrosis \\
\hline $0 \mathrm{mM}$ melittin & $93.50 \pm 1.20$ & $3.76 \pm 1.70$ & $1.43 \pm 0.10$ & $1.30 \pm 0.70$ \\
$0.7 \mathrm{mM}$ melittin & $90.75 \pm 0.15$ & $4.07 \pm 0.44$ & $2.94 \pm 0.12$ & $2.25 \pm 0.43$ \\
$2.5 \mathrm{mM}$ melittin & $73.70 \pm 3.60$ & $2.68 \pm 0.06$ & $13.05 \pm 1.15$ & $10.58 \pm 2.53$ \\
$0.9 \mathrm{mM}$ doxorubicin & $85.60 \pm 1.90$ & $9.08 \pm 2.52$ & $2.78 \pm 0.14$ & $2.50 \pm 0.76$ \\
\hline
\end{tabular}

Values are mean per cent of total cells \pm SEM, derived from three replications. 
(A)

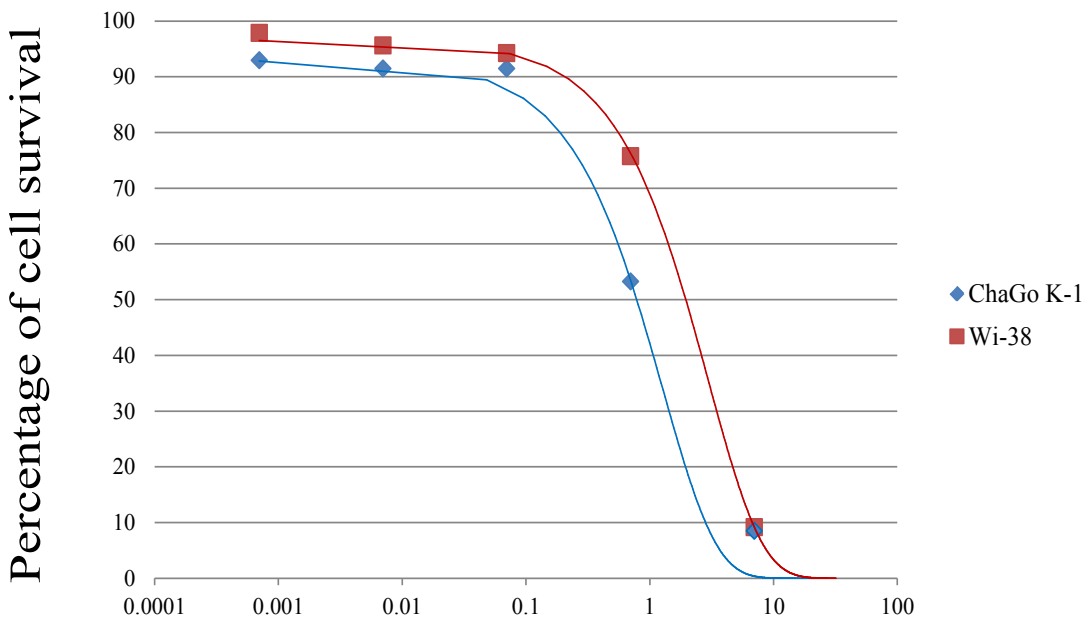

Melittin concentration $(\mu \mathrm{M})$

(B)

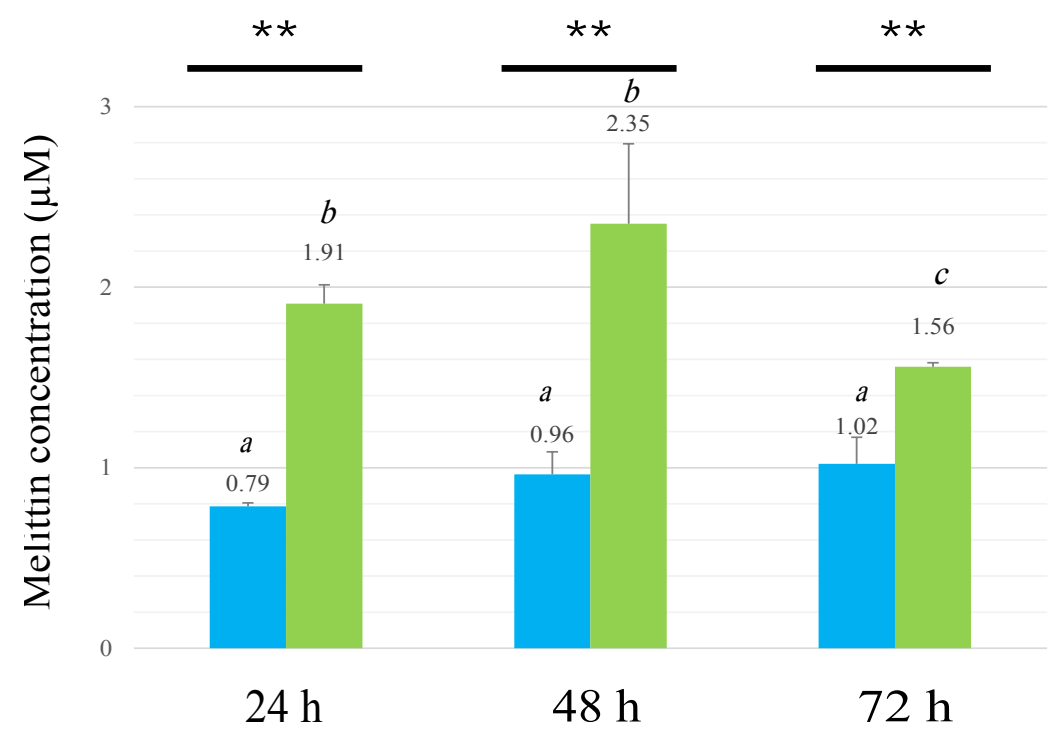

Figure 1. Effect of Melittin on ChaGo-K1 and Wi-38 Cell Viability. (A) The \% cell survival after a 24 h incubation period across different melittin concentrations. $* *$ indicates significant difference from the untreated group, $\mathrm{p}<0.01$. (B) The IC50 of melittin towards the ChaGo-K1 and Wi-38 cells after 24,48 , and $72 \mathrm{~h}$ incubation. ** indicates significant difference between cell types at the specific time point $(\mathrm{p}<0.01)$, and different letters indicate significantly different IC50 values $(\mathrm{p}<0.05)$.

was increased, although the trend was dose- and time-dependent (Figure 1B), with an $\mathrm{IC}_{50}$ for ChaGo-K1 and Wi-38 cells of $0.96 \pm 0.12$ and $2.35 \pm 0.44 \mu \mathrm{M}$, respectively, for the $48 \mathrm{~h}$ incubation and $1.02 \pm 0.15$ and $1.56 \pm 0.02 \mu \mathrm{M}$, respectively, for the $72 \mathrm{~h}$ incubation. The Wi-38 cells were less sensitive to melittin than the ChaGo-K1 cells at $48 \mathrm{~h}$ of incubation.

\section{Apoptosis in ChaGo-K1 cells}

To investigate the apoptotic effect of melittin on ChaGo-K1 cells, programmed cell death was analysed by flow cytometry. ChaGo-K1 cells were divided into four groups as viable cells (annexin V-FITC- PI-), early apoptotic cells (annexin V-FITC + PI-), late apoptotic cells (annexin V-FITC+ PI+), and necrotic cells (annexin V-FITC- PI+) using unstained cells (autofluorescence) as reference. The results showed that $0.7 \mu \mathrm{M}$ of melittin $\left(\mathrm{IC}_{50}\right)$ gave a similar trend as doxorubicin, which primarily increased the early apoptotic cells during the $24 \mathrm{~h}$ incubation period (Figure 2 and Table 1). However, $2.5 \mu \mathrm{M}$ of melittin $\left(\mathrm{IC}_{70}\right)$ increased the proportion of late apoptotic and necrotic cells (Figure 2 and Table 1).

\section{Cell cycle arrest by melittin}

Flow cytometric analysis of PI-stained cells revealed that the in vitro antiproliferative effect of melittin on ChaGo-K1 cells was likely to be caused by cell cycle arrest and different concentrations of melittin caused cell cycle arrest in different phases. Melittin at a concentration of $0.7 \mu \mathrm{M}\left(\mathrm{IC}_{50}\right)$ caused a slight cell cycle arrest at the $\mathrm{G} 2 / \mathrm{M}$ phase, and at $2.5 \mu \mathrm{M}\left(\mathrm{IC}_{70}\right)$, the cell cycle arrest was stronger and earlier at the $\mathrm{G} 0 / \mathrm{G} 1$ phase. On the other hand, doxorubicin, a currently used chemotherapeutic drug, caused a strong cell cycle arrest at the G2/M phase at $0.9 \mu \mathrm{M}\left(\mathrm{IC}_{50}\right)$ (Figure 3). These results support the inhibitory effect of melittin on the cell cycle progression 

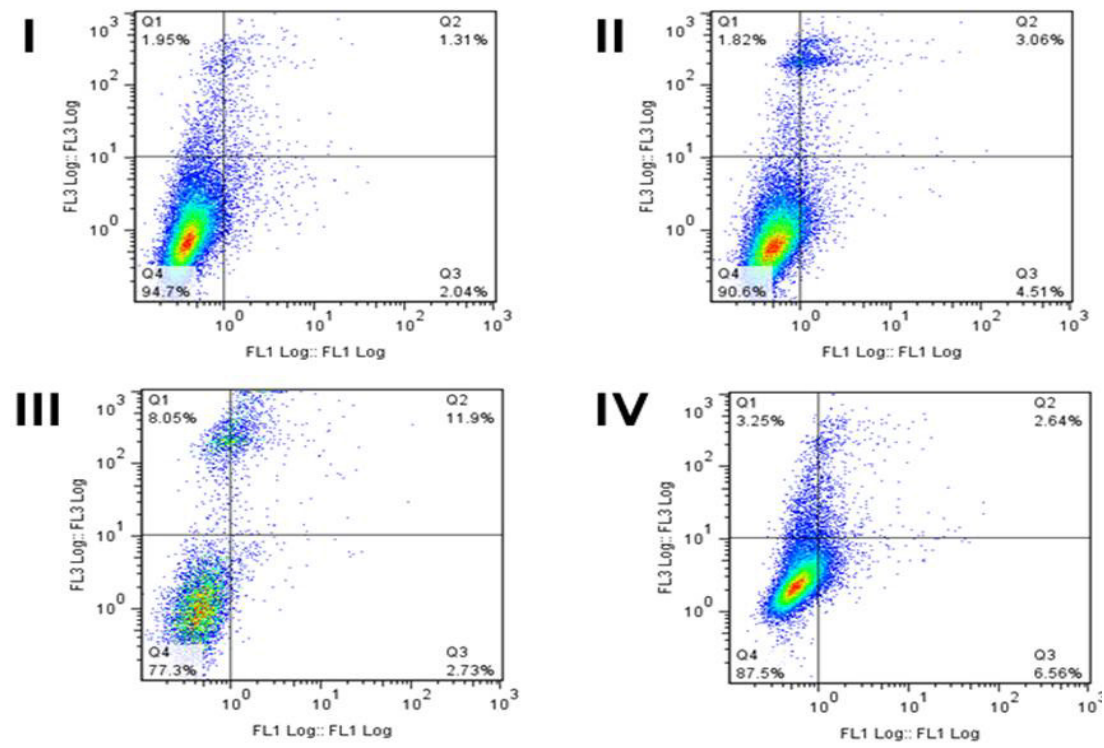

Figure 2. Programmed Cell Death of Melittin-Treated ChaGo-K1 Cells. Dot plot indicates annexin V-FITC (FL1) vs. PI staining (FL3) of cells treated with melittin at (I) $0 \mu \mathrm{M}$, (II) $0.7 \mu \mathrm{M}$, (III) $2.5 \mu \mathrm{M}$, and (IV) $0.9 \mu \mathrm{M}$ of doxorubicin after a $24 \mathrm{~h}$ incubation. Plots are representative of 3 independent repeats.

of ChaGo-K1 cells.

\section{Monocyte to macrophage differentiation}

The effect of melittin on IL-10 production by PMA-induced THP-1 and M2-polarized macrophages (induced with IL-4 and IL-13) was investigated. The production of IL-10 in THP-1-derived macrophages increased with increasing melittin concentrations, but was not statistically significant (Figure 4). IL-10 production by the M2-polarized macrophages was significantly greater (1.4-fold) than the THP-1 cells. However, melittin slightly inhibited the IL-10 production by the M2-polarized cells, and so potentially inhibited the differentiation of monocytes to M2 macrophages at the concentrations of 0.175 and $0.350 \mu \mathrm{M}$ (Figure 4).

Cell migration, angiogenesis, and changes in gene expression

Melittin could restrain the migration and colony formation of ChaGo-K1 (Figs. 5A) and Wi-38 cells (Fig. 5B). Although melittin at $\geq 1.4 \mu \mathrm{M}$ completely inhibited colony formation in both ChaGo-K1 and Wi-38 cells; at lower concentrations $(\geq 0.7 \mu \mathrm{M})$, it inhibited colony formation in ChaGo-K1 cells more than in Wi-38 cells, perhaps due to the smaller sized colonies formed by ChaGo-K1 cells.

Furthermore, it was found that melittin did not affect VEGF secretion or angiogenesis in ChaGo-K1 cells compared to untreated cells $(0.171$ and $0.191 \mathrm{ng} / \mathrm{mL}$, respectively). Cathepsin $\mathrm{S}$ plays an important role in cell proliferation, angiogenesis and metastasis (Gocheva et al., 2006), where an increased expression of CatS is always related to malignancies (Xu et al., 2009). The expression of CatS in the melittin-treated cells was not significantly higher than in the control (Figure 6). Thus, programmed cell death of melittin-treated ChaGo-K1 cells does not seem to be involved with angiogenesis and CatS expression. The expression levels of Bcl-2 and MADD,

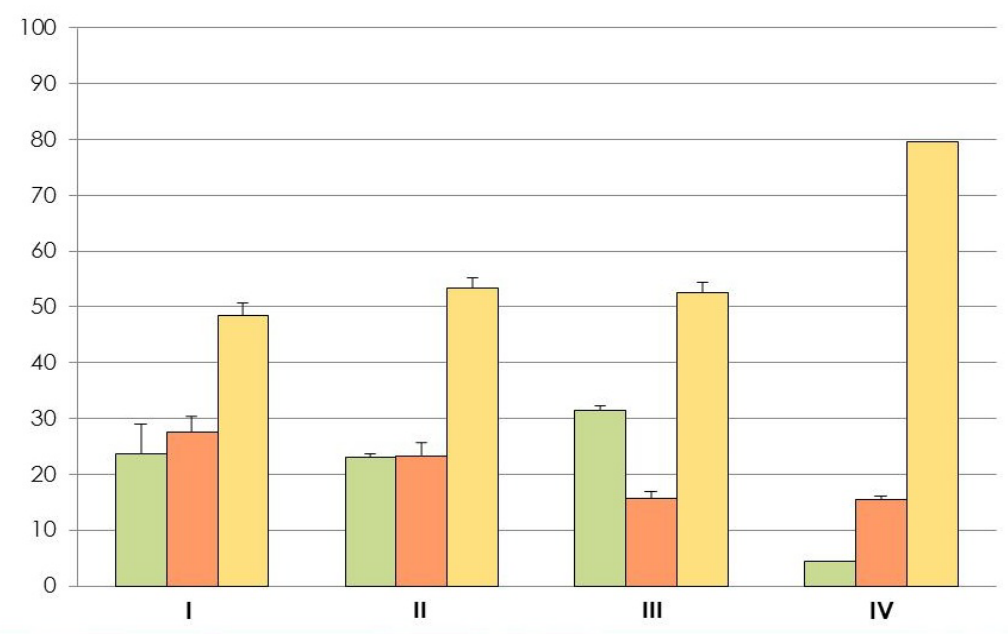

Figure 3. Percentage of ChaGo-K1 Cells in Each Phase of the Cell Cycle after treatment for 24 h with melittin at (I) 0 $\mu \mathrm{M}$, (II) $0.7 \mu \mathrm{M}$, (II) $2.5 \mu \mathrm{M}$, and (IV) $0.9 \mu \mathrm{M}$ of doxorubicin. Values are mean percent of total cells \pm SEM, derived from three replications. 


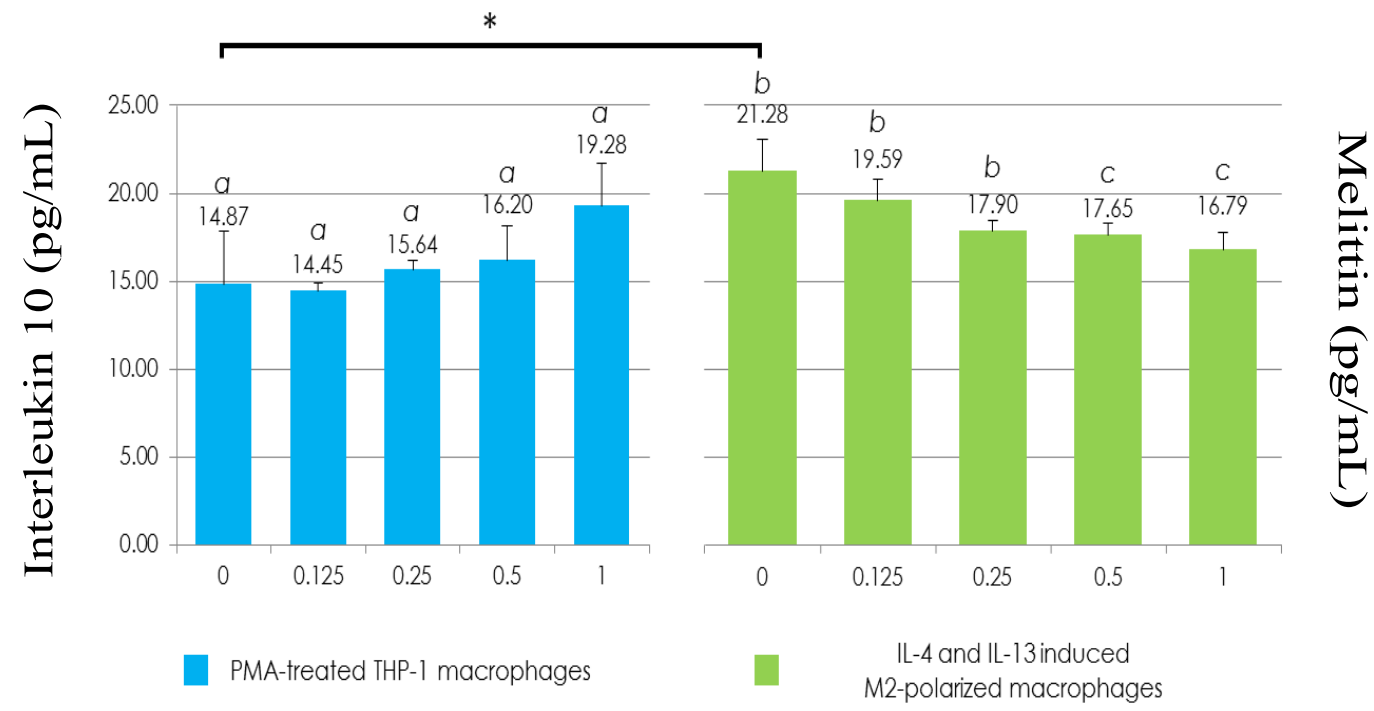

Figure 4. IL-10 Production in Melittin-Treated THP-1 Cells. The data for IL-10 production from PMA-induced macrophages (left, in blue) and IL-4 and IL-13-induced M2 polarized macrophages (right, in green). * represents a significant difference between the untreated groups of both macrophage types $(p<0.05)$, and different letters in each group represent significantly different IL-10 production $(\mathrm{p}<0.05)$.

as representative apoptotic genes, were evaluated (Figure 6). A significantly higher Bcl-2 and a lower MADD expression was observed in melittin-treated ChaGo-K1 cells, which supports the melittin-induced apoptosis observed by the annexin V-FITC/PI staining and flow cytometric analysis (Figurs 2 and 3 ).

\section{Discussion}

New chemotherapeutic compounds, especially from plants, have been used to treat cancer patients. However, side effects and drug-resistance occurs eventually.

In recent years, peptides and immunotherapy have emerged as other promising therapeutic approaches. Thus, melittin, was introduced here as a potential therapeutic agent for the treatment of lung cancers. It is beneficial in inducing early apoptosis in ChaGo-K1 cells after a $4 \mathrm{~h}$ exposure at the $\mathrm{IC}_{50}$ dose, which is earlier than cordycepin, isolated from Cordyceps sinensis, which took $48 \mathrm{~h}$ (Su et al., 2017). Furthermore, vglycin, a novel natural

(A)

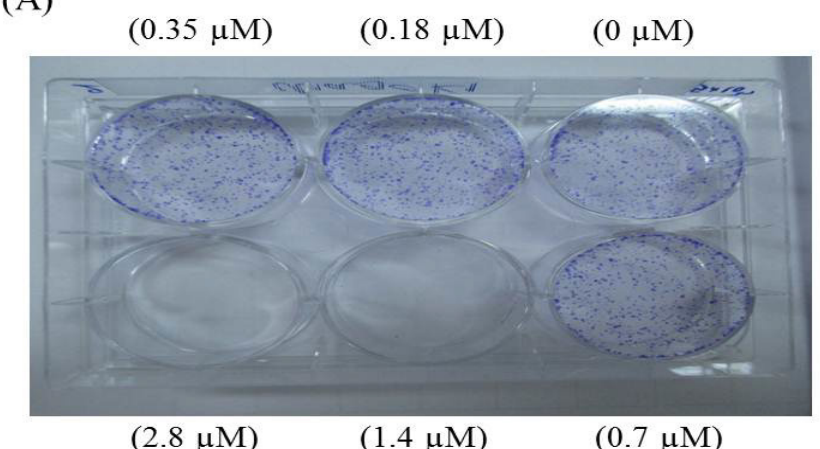

(B)

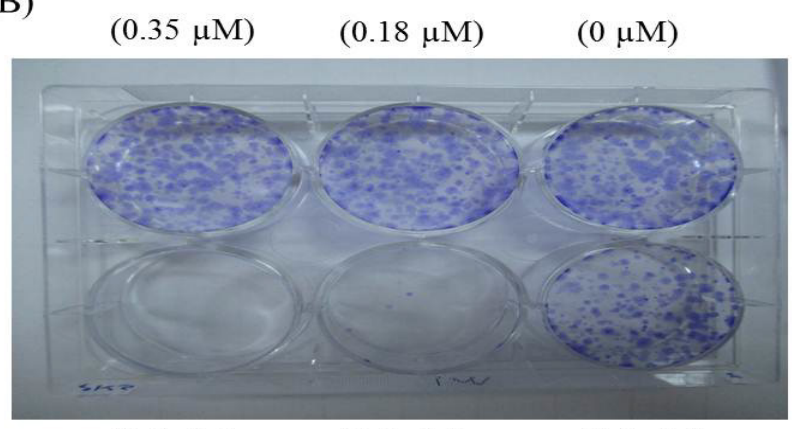
$(2.8 \mu \mathrm{M})$
$(1.4 \mu \mathrm{M})$
$(0.7 \mu \mathrm{M})$

Figure 5. Colony Formation. (A) ChaGo-K1 and (B) Wi-38 cells after $14 \mathrm{~d}$ of incubation in the presence of melittin at various concentrations $(0 \mu \mathrm{M}=$ control $)$ and stained with crystal violet. The magnification is $40 \times$. The figure is a representative of four replications. 


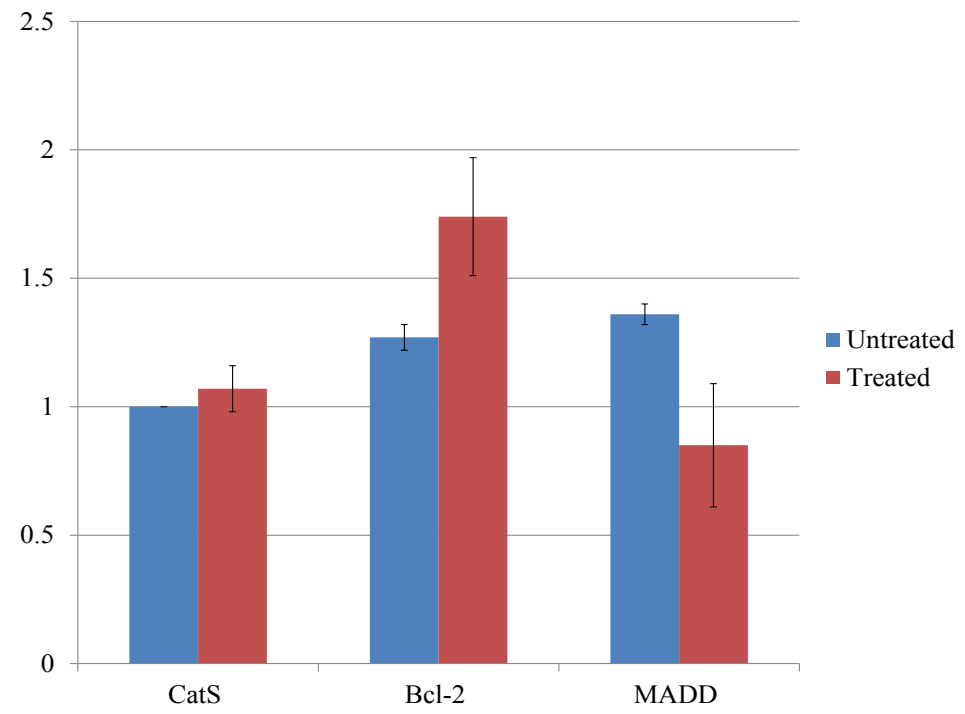

Figure 6. Change in Transcript Expression Levels of CatS and the Representative Apoptotic genes Bcl2 and MADD. ChaGo-K1 cells were cultured for $24 \mathrm{~h}$ in CM-R with $0 \mu \mathrm{M}$ (control) or $0.7 \mu \mathrm{M}$ melittin. Data are shown as mean $\pm 1 \mathrm{SD}$, derived from three independent repeats, and the relative expression levels are normalized to that of the housekeeping gene actin, used as control. Significant differences between the control and treated cells are shown at the $\mathrm{p}<0.05(*)$.

polypeptide isolated from pea seeds, took $24 \mathrm{~h}$ to cause a significant increase in apoptosis in the CT-26, SW480, and NCI-H716 colon cancer cell lines at $10 \mu \mathrm{M}$ (Gao et al., 2017).

Here, melittin-induced apoptosis of ChaGo-K1 was supported by the observed morphological changes (cell shrinkage, round cell formation, nucleus and organelle condensation, cell floating, decrease in viable cell density and presence of cell debris) and the significantly higher and lower expression of Bcl-2 and MADD transcripts, respectively. Similar altered gene expression was seen in apoptosis of breast cancer BT-474 cells induced by cardanol (Buahorm et al., 2015). However, whether melittin induced apoptosis or necrosis depended on the dose.

The tumour microenvironment is important for cancer survival and resistance. Hence, monocyte-to-macrophage differentiation was evaluated, focusing on the two distinct macrophage phenotypes of M1, which is involved in anti-tumour immunity, and the activated M2, which has pro-tumour properties. Ruffell et al. (2012) reported that M2-polarized macrophages promoted tumour growth and survival, and possibly caused cancer resistance. Thus, M2-polarized macrophages might be involved in TAMs. In this study, it is interesting that melittin inhibited M2 macrophage differentiation, but not THP-1. However, the molecular mechanism behind melittin inhibition awaits elucidation. It is possible that the M2 macrophages were reprogrammed to the M1 type (Yu et al., 2017), and so the expression of biomarkers for both M1 and M2 macrophages needs to be evaluated. Interestingly, ß-elemene, extracted from the Chinese herb Curcuma wenyujin, could induce the re-differentiation of M2 macrophages to the M1 type, where down-regulation of Arg-1 (M2 macrophage biomarker) and up-regulation of iNOS (M1 macrophage biomarker) was observed (Yu et al., 2017).
IL-10 is one of the major obstacles to immunotherapy, which reduces proliferation, cytokine production, and migratory capacities of effector T-cells (Dennis et al., 2013; Joyce and Fearon, 2015). Thus, localized inhibition of IL-10 could be a promising alternative co-treatment strategy. The combination of heterologous vaccination and cyclophosphamide was shown to cause immunopotentiation, in terms of a higher number of effector T-cells and tumour growth inhibition (Xia et al., 2016). In this study, melittin was found to decrease IL-10 production in M2 macrophages, but the underlying molecular mechanism is unknown.

In vitro colony formation is a product of both cell proliferation and migration. That melittin inhibited colony formation in both ChaGo-K1 and Wi-38 cells, suggests that its specificity to cancer cells was rather low.

In tumour cells and TAMs, CatS is produced to promote tumour growth, angiogenesis, migration, invasion, and metastasis (Fan et al., 2012; Sevenich et al., 2014). In this study, a non-significant higher expression of CatS was observed in melittin-treated ChaGo-K1 cells. Additionally, there was no significant change in the level of VEGF. It is possible that melittin affected ChaGo-K1 cells via cell migration but not the CatS and angiogenesis pathway. Furthermore, since there are many types of cathepsins, such as CatB and CatD that are involved in isocitrate dehydrogenase-wild type glioblastoma (Koh et al., 2017), and CatH in malignant prostate cancer (Jevnikar et al., 2013), the change in expression of those cathepsins should also be ascertained. For now, compounds synergistic with melittin, especially those that can inhibit CatS expression and angiogenesis, are of interest to inhibit ChaGo-K1 cells more effectively.

This study indicated that melittin could suppress ChaGo-K1 cell proliferation and cause apoptosis by upregulating Bcl-2 and down-regulating MADD expression. This suggests that melittin is a promising anti-lung cancer 
peptide. Future in vivo studies are required to determine the underlying molecular mechanisms.

\section{Acknowledgements}

This work was funded by Chulalongkorn University, the Ratchadapisek Sompoch Endowment Fund of Chulalongkorn University and Sci-Super IV_61_003.

\section{References}

Buahorm S, Puthong S, Palaga T, et al (2015). Cardanol isolated from Thai Apis mellifera propolis induces cell cycle arrest and apoptosis of BT-474 breast cancer cells via p21 upregulation. $D A R U, \mathbf{2 3}, 5$.

da Silva-Diz V, Lorenzo-Sanz L, Bernat-Peguera A, et al (2018). Cancer cell plasticity: Impact on tumour progression and therapy response. Semin Cancer Biol, doi: 10.1016/j. semcancer.2018.08.009.

Dennis KL, Blatner NR, Gounari F, et al (2013). Current status of IL-10 and regulatory T-cells in cancer. Curr Opin Oncol, 25, 637.

Fan Q, Wang X, Zhang H, et al (2012). Silencing cathepsin S gene expression inhibits growth, invasion, and angiogenesis of human hepatocellular carcinoma in vitro. Biochem Biophys Res Commun, 425, 703-10.

Felip E, Rosell R (2007). Testing for excision repair crosscomplementing 1 in patients with non- small-cell lung cancer for chemotherapy response. Expert Rev Mol Diagn, 7, 261-8.

Gajski G, Garaj-Vrhovac V (2013). Melittin: a lytic peptide with anticancer properties. Environ Toxicol Phar, 36, 697-705.

Gao C, Sun R, Xie YR, et al (2017). The soy-derived peptide Vglycin inhibits the growth of colon cancer cells in vitro and in vivo. Exp Biol Med, 242, 1034-43.

Gocheva V, Zeng W, Ke D, et al (2006). Distinct roles for cysteine cathepsin genes in multistage tumourigenesis. Gene Dev, 20, 543-56.

Hasan S, Renz P, Turrisi A, et al (2018). Dose escalation and associated predictors of survival with consolidative thoracic radiotherapy in extensive stage small cell lung cancer (SCLC): A National Cancer Database (NCDB) propensity-matched analysis. Lung Cancer, 124, 283-90.

Jablonski KA, Amici SA, Webb LM, et al (2015) Novel markers to delineate murine M1 and M2 macrophages. PLoS One, 10, e0145342.

Jevnikar Z, Rojnik M, Jamnik P, et al (2013). Cathepsin H mediates the processing of talin and regulates migration of prostate cancer cells. $J$ Biol Chem, 288, 2201-9.

Joyce JA, Fearon DT (2015). T cell exclusion, immune privilege, and the tumour microenvironment. Science, 348, 74-80.

Kerkar SP, Restifo NP (2012). Cellular constituents of immune escape within the tumour microenvironment. Cancer Res, 72, 3125-30.

Koh SP, Wickremesekera AC, Brasch HD, et al (2017). Expression of cathepsins B, D, and G in isocitrate dehydrogenase-wildtype glioblastoma. Front Surg, 4, 28.

Konrad CV, Murali R, Varghese BA, et al (2017). The role of cancer stem cells in tumor heterogeneity and resistance to therapy. Can J Physiol Pharmacol, 95, 1-15.

Liang SQ, Bührer ED, Berezowska S, et al (2018) mTOR mediates a mechanism of resistance to chemotherapy and defines a rational combination strategy to treat KRAS-mutant lung cancer. Oncogene, doi: 10.1038/s41388-018-0479-6.

Lin L, Zhu BP, Cai L (2017). Therapeutic effect of melittin on a rat model of chronic prostatitis induced by complete freund's adjuvant. Biomed Pharmacother, 90, 921-7.
Mohammadi S, Saghaeian-Jazi M, Sedighi S, et al (2017a). Immunomodulation in systemic lupus erythematosus: Induction of M2 population in monocyte-derived macrophages by pioglitazone. Lupus, 26, 1318-27.

Mohammadi S, Seyedhosseini FS, Behnampour N, et al (2017b). Indole-3-carbinol induces G1 cell cycle arrest and apoptosis through aryl hydrocarbon receptor in THP-1 monocytic cell line. J Recept Signal Transduct Res, 37, 506-14.

Pilling D, Galvis-Carvajal E, Karhadkar TR, et al (2017). Monocyte differentiation and macrophage priming are regulated differentially by pentraxins and their ligands. BMC Immunol, 18, 30.

Qin M, Wang L, Li F, et al (2017). Oxidized LDL activated eosinophil polarize macrophage phenotype from M2 to M1 through activation of CD36 scavenger receptor. Atherosclerosis, 263, 82-91.

Rady I, Siddiqui IA, Rady M, et al (2017). Melittin, a major peptide component of bee venom, and its conjugates in cancer therapy. Cancer Lett, 402, 16-31.

Ruffell B, Affara NI, Coussens LM (2012). Differential macrophage programming in the tumor microenvironment. Trends Immunol, 33, 119-26.

Sevenich L, Bowman RL, Mason SD, et al (2014). Analysis of tumor- and stroma-supplied proteolytic networks reveals a brain metastasis-promoting role for cathepsin S. Nat Cell Biol, 16, 876-88.

Shao LN, Zhu BS, Xing CG, et al (2016). Effects of autophagy regulation of tumor-associated macrophages on radiosensitivity of colorectal cancer cells. Mol Med Rep, 13, 2661-70.

Shi W, Li C, Li M, et al (2016). Antimicrobial peptide melittin against Xanthomonas oryzae pv. oryzae, the bacterial leaf blight pathogen in rice. Appl Microbiol Biotechnol, 100, 5059-67.

Thanee M, Yongvanit P, Loilome W (2012). Tumor microenvironment and its functions. Srinagarind Med J, 27, 424-32.

Torre LA, Bray F, Siegel RL, et al (2015). Global cancer statistics, 2012. CA Cancer J Clin, 65, 87-108.

Wong HS, Chang WC (2018). Losses of cytokines and chemokines are common genetic features of human cancers: the somatic copy number alterations are correlated with patient prognoses and therapeutic resistance. Oncoimmunology, 7, e1468951.

Xia Q, Geng F, Zhang FF, et al (2016). Enhancement of fibroblast activation protein $\alpha$-based vaccines and adenovirus boost immunity by cyclophosphamide through inhibiting IL-10 expression in 4T1 tumor bearing mice. Vaccine, 34, 4526-35.

$\mathrm{Xu} \mathrm{J}$, Li D, Ke Z, et al (2009). Cathepsin S is aberrantly overexpressed in human hepatocellular carcinoma. Mol Med Rep, 2, 713-8.

Yu X, Xu M, Li N, et al (2017). B-elemene inhibits tumor-promoting effect of M2 macrophage in lung cancer. Biochem Biophys Res Commun, 490, 514-20.

Zhang C, Gao L, Cai Y, et al (2016). Inhibition of tumor growth and metastasis by photoimmunotherapy targeting tumor-associated macrophage in a sorafenib-resistant tumor model. Biomaterials, 84, 1-12.

Zhang Z, Zhang H, Peng T, et al (2016). Melittin suppresses cathepsin $\mathrm{S}$-induced invasion and angiogenesis via blocking of the VEGF-A/VEGFR-2/MEK1/ERK1/2 pathway in human hepatocellular carcinoma. Oncol Lett, 11, 610-8.

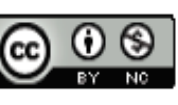

This work is licensed under a Creative Commons AttributionNon Commercial 4.0 International License. 